\title{
Non-invasive preimplantation genetic testing: a literature review
}

\author{
Larissa Nogueira Sousa ${ }^{1}$, Paula Bruno Monteiro ${ }^{1}$ \\ ${ }^{1}$ Centro Universitário Christus, Fortaleza, Ceará, Brazil
}

\begin{abstract}
Non-invasive preimplantation genetic testing emerged from the discovery of embryonic DNA in spent embryo culture medium. Considering that such methodology would be an important advance in assisted reproduction, this study aimed to evaluate the current scientific evidence, based on the reliability of non-invasive chromosome screening, through a literature review. We analyzed 14 original research papers in PubMed and SciELO, in English and Portuguese, published between 2016 and 2021 related to the topic. The agreement rate for ploidy compared to the traditional method ranged from 3.5\% - 93.8\% raising the discussion about the possible causes of this large variation, which may be due to the day of collection, spent culture media contamination, amplification methodology or the cytogenetic method used by each author. We concluded that the non-invasive test has many advantages over the traditional method, but that clinical replacement is not yet possible, and further studies are needed in order to have an accurate clinical test with the non-invasive methodology.
\end{abstract}

Keywords: biopsy, genetic testing, aneuploidy, assisted reproduction

\section{INTRODUCTION}

Preimplantation genetic testing (PGT) is defined as the multidisciplinary clinical application of genetic technologies and assisted reproduction techniques aimed at examining cells from an embryo in its in vitro developmental phase (Zegers-Hochschild et al., 2017). Such techniques began to be used for genetic screening, with the purpose of trying to improve pregnancy rates in certain groups of patients with poor IVF-procedure prognosis, such as those with advanced maternal age, recurrent miscarriages and repeated deployment failures. The test is done through an embryo biopsy, and one can remove the polar bodies, blastomere or trophectoderm (Almeida et al., 2013; Soeiro, 2012).

The trophectoderm biopsy is the most used, as it can be performed from the fifth day of embryonic development - blastocyst stage, in which the embryo is more developed, thus having more material for analysis, yielding more precision to the result (Homer, 2019). However, it is an invasive procedure, and it may compromise embryo viability and implantation potential, and it adds potential concerns about long-term effects on the offspring, which are very difficult to assess. In addition, the procedure for performing the biopsy requires considerable training and experience to manipulate the embryo, increasing the costs of performing the technique (Xu et al., 2016).

Thus, with the discovery of free embryonic DNA (cfeDNA) found in both blastocele fluid (BF) and spent culture medium (SCM) of the embryo, the possibility of performing a non-invasive preimplantation genetic test (NiPGT), eliminating the need for embryo biopsy, avoiding the risks related to the invasive procedure, with a simpler and potentially more affordable methodology, in addition to enabling the test in embryos with non-viable morphology for biopsy, with a better representation of the entire embryo.
Currently, there are two main research approaches for collecting cfeDNA for aneuploidy testing, they are: collection of spent culture medium, and combining BF collection with spent culture medium (Kuznyetsov et al., 2018; Stigliani et al., 2014).

Considering that the NiPGT methodology would be an important advance in assisted reproduction, this study aimed to evaluate the current scientific evidence based on the reliability of non-invasive chromosome screening, using extra embryonic DNA and its clinical potential.

\section{MATERIALS AND METHODS}

This is a literature review, aimed to address the techniques of invasive and non-invasive preimplantation genetic testing methods, evaluating the advantages and disadvantages of both techniques, bringing together current studies on advances in non-invasive testing, highlighting the level of reliability and its clinical potential. Data collection began in August 2020 and was carried out until May 2021, in the scientific databases PubMed and SciELO (Figure 1), using the keywords: biopsy, genetic testing, aneuploidy and assisted reproduction. We found 1,100 papers and analyzed 12 original articles that assess the NiPGT methodology, published between 2015 and 2021, in English and Portuguese. Tests using animals, monographs, case reports and review articles were not part of the results. The analyzed data were organized in a table using the Microsoft Excel ${ }^{\odot}$ platform.

\section{RESULTS}

Among the 12 studies analyzed (Table 1), only Feichtinger et al. (2017) did not use trophectoderm biopsy to validate their experiment. The analysis was performed by comparing spent culture media with polar body biopsy, which brought limitations to the analysis, such as lack of paternal chromosomal information. While the other authors used analytes in addition to trophectoderm biopsy ( $\mathrm{Ku}$ znyetsov et al., 2018; Ho et al., 2018; Huang et al., 2019; Yin et al., 2021; Rubio et al., 2019; Yeung et al., 2019; Jiao et al., 2019), such as blastocele fluid (Kuznyetsov et al., 2018; Jiao et al., 2019) and whole embryo (Xu et al., 2016; Kuznyetsov et al., 2018; Ho et al., 2018; Huang et al., 2019). The agreement rate between spent culture medium (SCM) and polar body biopsy was $27 \%$, lower than the SCM analysis with blastocele fluid, which achieved an agreement of $87.5 \%$ (Kuznyetsov et al., 2018). For those who chose to correlate the spent culture medium with the trophectoderm biopsy, the agreement rate ranged from $3.5 \%-93.8 \%$ raising the discussion about the possible causes for this large variation, which may be due to the day of collection of spent culture media, media contamination, amplification methodology or cytogenetic method used by each author.

The choice to collect day 3 and day 5/6 developmental culture medium was because this is a convenient time point in the laboratory evaluation of embryos. Analysis of the two-stage culture medium was performed by $75 \%$ of the studies, while the other authors used a single-stage culture medium (Feichtinger et al., 2017; Liu et al., 2017; Ho et al., 2018). Regarding the methods of analysis of the 
Table 1. Studies that used spent culture medium to validate NiPGT.

\begin{tabular}{|c|c|c|c|c|c|c|}
\hline & \multicolumn{3}{|c|}{ Samples } & \multicolumn{3}{|c|}{ Analyze } \\
\hline & $\begin{array}{c}\text { Development } \\
\text { Day }\end{array}$ & $\begin{array}{l}\text { Number } \\
\text { of } \\
\text { samples }\end{array}$ & $\begin{array}{l}\text { culture } \\
\text { medium }\end{array}$ & Agreement & $\begin{array}{c}\text { Amplification } \\
\text { Kits }\end{array}$ & $\begin{array}{l}\text { Cytogenetics } \\
\text { Method }\end{array}$ \\
\hline $\begin{array}{l}\text { Spent culture medium } \\
\text { Shamonki et al. (2016) } \\
\text { Xu et al. (2016) } \\
\text { Feichtinger et al. (2017) } \\
\text { Liu et al. (2017) } \\
\text { Ho et al. (2018) } \\
\text { Huang et al. (2019) } \\
\text { Vera-Rodriguez et al. (2018) } \\
\text { Yin et al. (2021) } \\
\text { Rubio et al. (2019) } \\
\text { Yeung et al. (2019) }\end{array}$ & $\begin{array}{l}\text { D3-5/6 } \\
\text { D3-5 } \\
\text { D1-5 } \\
\text { D1-5 } \\
\text { D3-5 } \\
\text { D5/6 } \\
\text { D3-5 } \\
\text { D5 } \\
\text { D4-5/6/7 } \\
\text { D3-5/6 }\end{array}$ & $\begin{array}{c}57 \\
42 \\
22 \\
88 \\
41 \\
52 \\
56 \\
75 \\
115 \\
168\end{array}$ & $\begin{array}{l}\text { two steps } \\
\text { two steps } \\
\text { single step } \\
\text { single step } \\
\text { single step } \\
\text { two steps } \\
\text { two steps } \\
\text { two steps } \\
\text { two steps } \\
\text { two steps }\end{array}$ & $\begin{array}{c}3.5 \% \\
85.7 \% \\
27 \% \\
64.5 \% \\
\text { D3-56.3\%/D5-65\% } \\
93.8 \% \\
30.4 \% \\
27.2 \% \\
\text { D5-78.7\%/D6/7- 84\% } \\
62.1 \%\end{array}$ & $\begin{array}{c}\text { Repli-G } \\
\text { MALBAC } \\
\text { SurePlex } \\
\text { MALBAC } \\
\text { Picoplex } \\
\text { MALBAC } \\
\text { SurePlex } \\
\text { ChromInst } \\
\text { ThermoFisher } \\
\text { SurePlex }\end{array}$ & $\begin{array}{l}\text { CGH } \\
\text { NGS } \\
\text { CGH } \\
\text { NGS } \\
\text { NGS } \\
\text { NGS } \\
\text { CGH } \\
\text { NGS } \\
\text { NGS } \\
\text { NGS }\end{array}$ \\
\hline $\begin{array}{l}\text { Spent culture medium and } \\
\text { blastocoel fluid } \\
\text { Kuznyetsov et al. (2018) } \\
\text { Jiao et al. (2019) }\end{array}$ & $\begin{array}{c}\text { D5-6/7 } \\
\text { D5/6 }\end{array}$ & $\begin{array}{l}47 \\
62\end{array}$ & $\begin{array}{l}\text { two steps } \\
\text { two steps }\end{array}$ & $\begin{array}{l}87.5 \% \\
76.1 \%\end{array}$ & $\begin{array}{l}\text { SurePlex } \\
\text { MALBAC }\end{array}$ & $\begin{array}{l}\text { NGS } \\
\text { NGS }\end{array}$ \\
\hline
\end{tabular}

Main studies reporting the use of spent culture medium for preimplantation genetic testing. D1-7, day of embryonic development; NGS, next-generation sequencing; $\mathrm{CGH}$, comparative genomic hybridization.

collected material, SCM, trophectoderm biopsy and of polar bodies went through the same protocol. Of the selected studies, 33\% used Sureplex as a method of DNA amplification. For chromosomal analysis, the most used cytogenetic method was Next Generation Sequencing (NGS).

\section{DISCUSSION}

Hammond et al. (2016) demonstrated higher levels of DNA in culture media that were exposed to embryos compared to culture media controls, suggesting that much of the genetic material detected in spent culture media is of embryonic origin. From this discovery, emerged studies and variations regarding the collection of SCM.

The length of time an embryo maintains contact with the enriched medium depends on the standard operating procedures of the IVF laboratory. While some laboratories cultivate embryos in a single-stage, monophasic medium (embryo is cultured in the same drop in the middle from day 1 to day $5 / 6)$, others chose to sequentially change the culture medium once or twice between fertilization and blastulation (Wu et al., 2015).

Studies using single-stage culture medium hypothesized that this protocol could increase the level of excreted DNA, due to greater exposure of the embryo in the SCM. Feichtinger et al. (2017) and Liu et al. (2017) demonstrated amplification rates of samples from the culture medium of $81.8 \%$ and $90.9 \%$, with an average DNA yield of 21.33 $\mathrm{ng} / \mu \mathrm{l}$ and $25 \mathrm{ng} / \mu \mathrm{l}$, respectively. However, cfDNA degradation may increase over time, decreasing cfDNA quality (Feichtinger et al., 2017; Liu et al., 2017; Ho et al., 2018).

Shamonki et al. (2016) and other authors (Xu et al., 2016; Kuznyetsov et al., 2018; Huang et al., 2019; Vera-Rodriguez et al., 2018; Yin et al., 2021; Rubio et al., 2019; Yeung et al., 2019; Jiao et al., 2019) used the methodology of embryo cultivation in a two-stage media, pointing out a hypothesis that the exchange of culture media would decrease the rate of maternal contamination and the degradation of embryonic DNA. Rubio et al. (2019) found in their study that the longer the embryo remains in specific culture conditions, the greater the specificity of the test, with no significant impact on sensitivity. This statement came from their results that showed that the false positive (FP) and false negative (FN) values were better when considering day $6 / 7$ of the SCM, with only $8.6 \%$ FP and $2.5 \% \mathrm{FN}$ and increase sensitivity and specificity of $95.2 \%$ and $82.1 \%$, respectively; compared to the analysis made on day $4 / 5$ of the SMC, where $13.9 \%$ were FP and $2.8 \%$ $\mathrm{FN}$, and the sensitivity and specificity were $94.5 \%$ and $71.7 \%$, respectively.

It is extremely important to consider how the embryo is treated during its development, because it can determine not only the quantity and quality of cfDNA, but also, and more importantly, its origin. In addition to nuclear DNA, mitochondrial DNA (mtDNA) can also be detected in the embryo's culture medium (Stigliani et al., 2013; Wu et al., 2015). However, the secretion mechanism of this DNA is not fully understood. The hypothesis is that this genetic material can be released from cells undergoing apoptosis as part of a controlled elimination process. It is believed that the DNA of spent culture medium comes from both the inner cell mass (ICM) and the trophectoderm (TE) because both strains undergo apoptosis during pre-implantation development (Kuznyetsov et al., 2018; Ho et al., 2018; Wu et al., 2015).

The hypothesis that the DNA comes from the entire embryo brings advantages to NIPGT over the pre-implantation genetic test currently used, as although there seems to be a high rate of agreement between TE cells and ICM cells, a TE biopsy may not always represent the whole embryo(Kuznyetsov et al., 2018; McCoy, 2017). Studies that used the whole embryo as the analyte showed agreement rates with NiPGT ranging from $56.3 \%-96.6 \%$, the authors point out maternal contamination and embryo mosaicism as possible causes for this variability (Xu et al., 2016; Kuznyetsov et al., 2018; Ho et al., 2018; Huang et al., 2019).

Embryonic mosaicism is one of the main limitations of the PGT. It is defined as mitotic errors that appear in embryonic cells after the cleavage stage and form a mixture of euploid and aneuploid cells that can present in different ways, and when detected there is debate about embryo transfer or disposal (McCoy, 2017). Transfers can result in successful implantation and healthy births (Eggenhuizen et al., 2021; Greco et al., 2015), but it can also result in decreased implantation, as well as an increased risk of genetic abnormalities and adverse pregnancy outcomes (Eggenhuizen et al., 2021). 


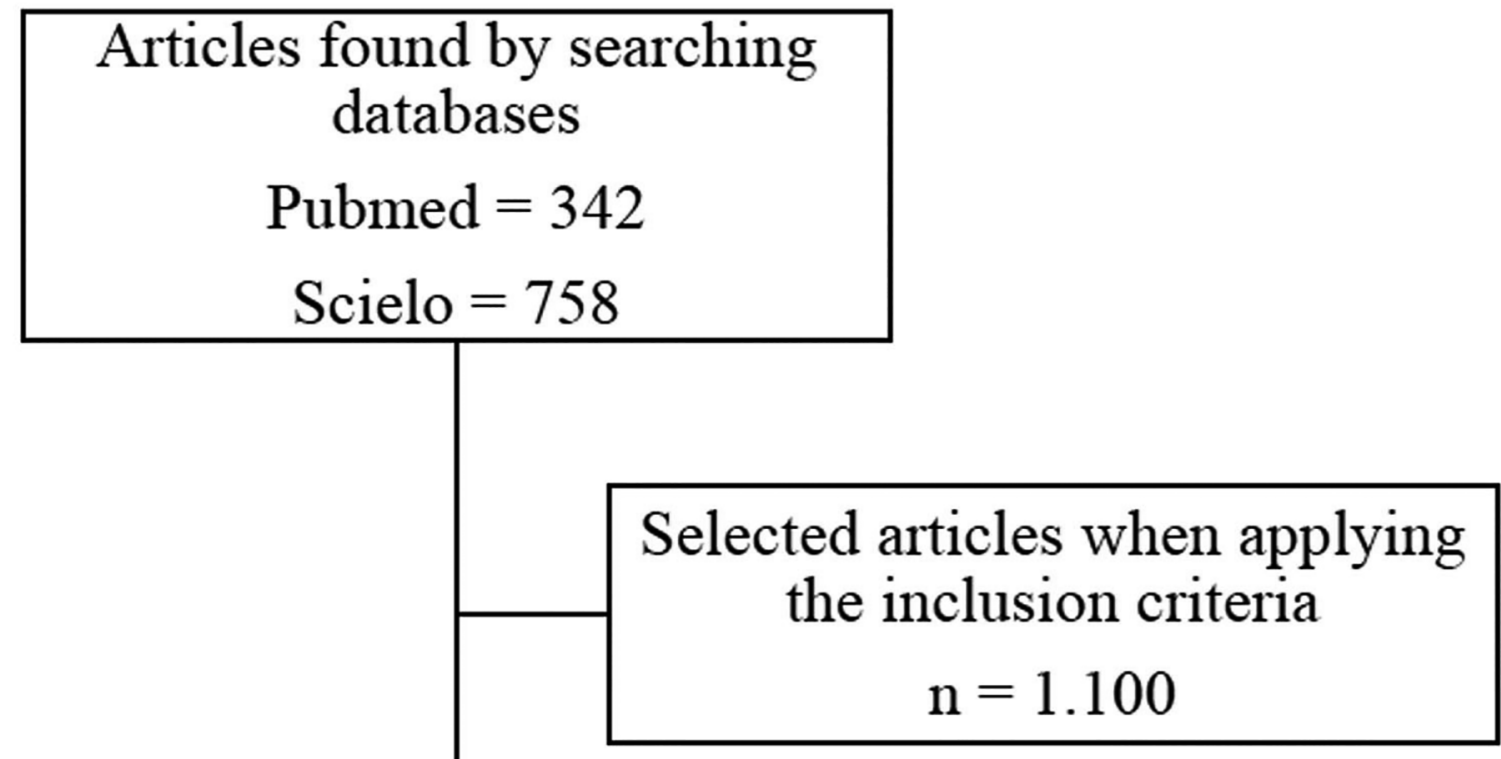

\begin{tabular}{|}
$\begin{array}{l}\text { Articles excluded when the } \\
\text { exclusion criteria applied } \\
\mathrm{n}=1.088\end{array}$ \\
\hline
\end{tabular}

Another point to consider is the risk that the genetic material detected in the spent culture medium is due to contamination, which can occur for several reasons, but the most discussed is the maternal contamination that can arise from cumulus cells that remain adhered to the zona pellucida after denudation, or the polar bodies after their extrusion from the oocyte (Hammond et al., 2016). An analysis using single nucleotide polymorphism (SNP) was performed to determine the ratio between embryonic and maternal DNA. The group used the alleles identified in the trophectoderm biopsies as a reference for the embryonic DNA haplotype; and, as a reference for maternal contamination they used alleles identified in the follicular fluid. The rate of partial and total maternal contamination was $60.8 \%$ (Vera-Rodriguez et al., 2018). To prevent this possible contamination, the authors suggest that cumulus cells be carefully washed (Xu et al., 2016; Huang et al., 2019).
Yeung et al. (2019) included in their experiment an additional wash on day 3 , before each embryo was transferred to its individual culture. While Jiao et al. (2019) demonstrated minimal or no maternal contamination, through the hypothesis that the use of cryopreserved blastocysts, due to thawing and variation in the concentration of reagents, helped eliminate cumulus cells.

Different whole genome amplification (WGA) protocols seem to affect cfDNA amplification success rates in SCM samples. The two methodologies most present in the studies are the Surelex/PicoPlex and Multi-Loop Based Amplification Cycles (MALBAC), which promise less amplification bias compared to SurePlex. According to a study in which the two techniques were compared, SurePlex proved to be more suitable for detecting changes in copy number than MALBAC, in which the amplified samples showed non-uniformity across the genome, leading to false positives (Deleye et al., 2015). 
Studies have shown that modifications were needed in both types of methodology, such as the addition of six non-standard Sureplex amplification cycles, to ensure that the amount of DNA was sufficient (Ho et al., 2018). Improvements in MALBAC, which reduced the number of steps for library preparation of new primer designs from ten hours to two and a half hours, yielding high quality read rates (Jiao et al., 2019).

Regarding pre-implantation genetic screening methods, next-generation sequencing (NGS) and comparative genomic hybridization (CGH) are the most used, as they enable a complete genome analysis. NGS excels due to robust, high-throughput, customizable parallel analysis of multiple samples in a single sequencing run. A study comparing the two techniques using trophectoderm biopsy cells as samples provided clinical evidence that NGS detected all types of human blastocyst aneuploidies, segmental changes suggesting detection of partial aneuploidies or unbalanced translocations and mosaicism more accurately compared to mosaicism in the CGH screening (Yang et al., 2015).

A recent study compared the two chromosomal analysis techniques Veriseq (Illumina ${ }^{\circledR}$ ) and NICS (Yikon ${ }^{\circledR}$ ) when used in SCM, raising the question whether the disagreements in the NIPGT results are due to the techniques used. The results led to a similar diagnostic agreement $(74.6 \%$ SCM-NICS vs. $72.3 \%$ SCM-Veriseq), suggesting that the disagreements are not due to technical limitations. As for the SCM sensitivity analyses, both techniques (78.0 for NICS and 80.0 for Veriseq) were higher than the specificity (69.7 for NICS and 60.6 for Veriseq); therefore, the authors suggest that the method is more effective in identifying embryos with chromosomal abnormalities than in selecting normal and transferable embryos (Lledo et al., 2021). Sensitivity values were similar to the studies selected in this review, which ranged from $73.3 \%$ to $95.2 \%$ (Xu et al., 2016; Feichtinger et al., 2017; Ho et al., 2018; Vera-Rodriguez et al., 2018; Yeung et al., 2019).

\section{CONCLUSION}

The results found report successful amplification, high agreement rates and good sensitivity and specificity of the culture medium analysis. We can conclude that the time the embryo remains in contact with the culture medium, the need for complete removal of cumulus cells and the combination of blastocele fluid with spent culture medium favored the results.

However, NiPGT does not yet have enough reliability to replace PGT in assisted reproduction clinics, but it has many advantages when compared to the traditional invasive method, such as the easy methodology without the need for embryo manipulation and the screening of embryos that do not have viable morphology for biopsy. In order to elucidate all the issues raised in this study, it is necessary to agree on the chromosomal amplification and analysis methodology used, in order to define the underlying causes of the disagreement with the results of the embryo biopsy samples. In addition to standardizing protocols for media collection. More studies should be carried out using larger samples, so that we can better understand the mechanisms of DNA origin and release in the medium.

\section{CONFLICT OF INTEREST}

The authors declare no conflict of interest.

\section{Corresponding author:}

Larissa Nogueira Sousa

Centro Universitario Christus

Fortaleza, Ceará, Brazil

E-mail: nslarissa08@hotmail.com

\section{REFERENCES}

Almeida PBL, Duarte Filho OB, Soares JB. Prospects of using comparative genomic hybridization in blastocyst stage human embryo biopsy as pre-implantation genetic screening. Repr Clim. 2013;28:74-9. DOI: 10.1016/j.recli.2013.05.055

Deleye L, De Coninck D, Christodoulou C, Sante T, Dheedene A, Heindryckx B, Van den Abbeel E, De Sutter P, Menten B, Deforce D, Van Nieuwerburgh F. Whole genome amplification with SurePlex results in better copy number alteration detection using sequencing data compared to the MALBAC method. Sci Rep. 2015;5:11711. PMID: 26122179 DOI: $10.1038 /$ srep 11711

Eggenhuizen GM, Go A, Koster MPH, Baart EB, Galjaard RJ. Confined placental mosaicism and the association with pregnancy outcome and fetal growth: a review of the literature. Hum Reprod Update. 2021;27:885-903. PMID: 33984128 DOI: $10.1093 /$ humupd/dmab009

Feichtinger M, Vaccari E, Carli L, Wallner E, Mädel U, Figl K, Palini S, Feichtinger W. Non-invasive preimplantation genetic screening using array comparative genomic hybridization on spent culture media: a proof-of-concept pilot study. Reprod Biomed Online. 2017;34:583-9. DOI: 10.1016/j.rbmo.2017.03.015 PMID: 28416168 DOI: PMID: 28416168 DOI: $10.1016 /$ j.rbmo.2017.03.015

Greco E, Minasi MG, Fiorentino F. Healthy Babies after Intrauterine Transfer of Mosaic Aneuploid Blastocysts. N Engl J Med. 2015;373:2089-90. PMID: 26581010 DOI: 10.1056/NEJMc1500421

Hammond ER, Shelling AN, Cree LM. Nuclear and mitochondrial DNA in blastocoele fluid and embryo culture medium: evidence and potential clinical use. Hum Reprod. 2016;31:1653-61. PMID: 27270971 DOI: 10.1093/humrep/dew 132

Ho JR, Arrach N, Rhodes-Long K, Ahmady A, Ingles S, Chung K, Bendikson KA, Paulson RJ, McGinnis LK. Pushing the limits of detection: investigation of cell-free DNA for aneuploidy screening in embryos. Fertil Steril. 2018;110:467-75.e2. PMID: 29960707 DOI: 10.1016/j. fertnstert.2018.03.036

Homer HA. Preimplantation genetic testing for aneuploidy (PGT-A): The biology, the technology and the clinical outcomes. Aust N Z J Obstet Gynaecol. 2019;59:317-24. PMID: 30811595 DOI: 10.1111/ajo.12960

Huang L, Bogale B, Tang Y, Lu S, Xie XS, Racowsky C. Noninvasive preimplantation genetic testing for aneuploidy in spent medium may be more reliable than trophectoderm biopsy. Proc Natl Acad Sci U S A. 2019;116:14105-12. PMID: 31235575 DOI: 10.1073/pnas.1907472116

Jiao J, Shi B, Sagnelli M, Yang D, Yao Y, Li W, Shao L, Lu S, Li D, Wang $X$. Minimally invasive preimplantation genetic testing using blastocyst culture medium. Hum Reprod. 2019;34:136979. PMID: 31251795 DOI: 10.1093/humrep/dez075

Kuznyetsov V, Madjunkova S, Antes R, Abramov R, Motamedi G, Ibarrientos Z, Librach C. Evaluation of a novel non-invasive preimplantation genetic screening approach. PLoS One. 2018;13:e0197262. PMID: 29746572 DOI: $10.1371 /$ journal. pone.0197262 
Liu W, Liu J, Du H, Ling J, Sun X, Chen D. Non-invasive pre-implantation aneuploidy screening and diagnosis of beta thalassemia IVSII654 mutation using spent embryo culture medium. Ann Med. 2017;49:319-28. DOI: 10.1080/07853890.2016.1254816 PMID: 27786563 DOI: $10.1080 / 07853890.2016 .1254816$

Lledo B, Morales R, Ortiz JA, Rodriguez-Arnedo A, Ten J, Castillo JC, Bernabeu A, Llacer J, Bernabeu R. Consistent results of non-invasive PGT-A of human embryos using two different techniques for chromosomal analysis. Reprod Biomed Online. 2021;42:555-63. DOI: $10.1016 / \mathrm{j}$. rbmo.2020.10.021 PMID: 33454211 DOI: $10.1016 / j$. rbmo.2020.10.021

McCoy RC. Mosaicism in Preimplantation Human Embryos: When Chromosomal Abnormalities Are the Norm. Trends Genet. 2017;33:448-63. PMID: 28457629 DOI: 10.1016/j. tig.2017.04.001

Rubio C, Rienzi L, Navarro-Sánchez L, Cimadomo D, García-Pascual CM, Albricci L, Soscia D, Valbuena D, Capalbo A, Ubaldi F, Simón C. Embryonic cell-free DNA versus trophectoderm biopsy for aneuploidy testing: concordance rate and clinical implications. Fertil Steril. 2019;112:510-9. PMID: 31200971 DOI: 10.1016/j.fertnstert.2019.04.038

Shamonki MI, Jin H, Haimowitz Z, Liu L. Proof of concept: preimplantation genetic screening without embryo biopsy through analysis of cell-free DNA in spent embryo culture media. Fertil Steril. 2016;106:1312-8. PMID: 27565258 DOI: $10.1016 /$ j.fertnstert.2016.07.1112

Soeiro CASP. Preimplantation Genetic Diagnosis [Master's these]. Porto: Faculty of Medicine, University of Porto; 2012. Available at: https://repositorio-aberto.up.pt/bitstream/10216/72009/2/29084.pdf

Stigliani S, Anserini P, Venturini PL, Scaruffi P. Mitochondrial DNA content in embryo culture medium is significantly associated with human embryo fragmentation. Hum Reprod. 2013;28:2652-60. PMID: 23887072 DOI: 10.1093/ humrep/det314

Stigliani S, Persico L, Lagazio C, Anserini P, Venturini PL, Scaruffi P. Mitochondrial DNA in Day 3 embryo culture medium is a novel, non-invasive biomarker of blastocyst potential and implantation outcome. Mol Hum Reprod. 2014;20:1238-46. PMID: 25232043 DOI: 10.1093/ molehr/gau086
Vera-Rodriguez M, Diez-Juan A, Jimenez-Almazan J, Martinez S, Navarro R, Peinado V, Mercader A, Meseguer M, Blesa D, Moreno I, Valbuena D, Rubio C, Simon C. Origin and composition of cell-free DNA in spent medium from human embryo culture during preimplantation development. Hum Reprod. 2018;33:745-56. DOI: 10.1093/humrep/dey028 PMID: 29471395 DOI: 10.1093/humrep/dey028

Wu H, Ding C, Shen X, Wang J, Li R, Cai B, Xu Y, Zhong $Y$, Zhou C. Medium-based noninvasive preimplantation genetic diagnosis for human a-thalassemias-SEA. Medicine (Baltimore). 2015;94:e669. PMID: 25816038 DOI: 10.1097/MD. 0000000000000669

Xu J, Fang R, Chen L, Chen D, Xiao JP, Yang W, Wang $H$, Song $X$, Ma T, Bo S, Shi C, Ren J, Huang L, Cai LY, Yao B, Xie XS, Lu S. Noninvasive chromosome screening of human embryos by genome sequencing of embryo culture medium for in vitro fertilization. Proc Natl Acad Sci U S A. 2016;113:11907-12. PMID: 27688762 DOI: 10.1073/ pnas. 1613294113

Yang Z, Lin J, Zhang J, Fong WI, Li P, Zhao R, Liu X, Podevin $W$, Kuang $Y$, Liu J. Randomized comparison of next-generation sequencing and array comparative genomic hybridization for preimplantation genetic screening: a pilot study. BMC Med Genomics. 2015;8:30. DOI: 10.1186/s12920015-0110-4 PMID: 26100406 DOI: 10.1186/s12920-0150110-4

Yeung QSY, Zhang YX, Chung JPW, Lui WT, Kwok YKY, Gui B, Kong GWS, Cao Y, Li TC, Choy KW. A prospective study of non-invasive preimplantation genetic testing for aneuploidies (NiPGT-A) using next-generation sequencing (NGS) on spent culture media (SCM). J Assist Reprod Genet. $2019 ; 36: 1609-21$. DOI: $10.1007 / \mathrm{s} 10815-019-01517-$ 7 PMID: 31292818 DOI: 10.1007/s10815-019-01517-7

Yin B, Zhang H, Xie J, Wei Y, Zhang C, Meng L. Validation of preimplantation genetic tests for aneuploidy (PGT-A) with DNA from spent culture media (SCM): concordance assessment and implication. Reprod Biol Endocrinol. 2021;19:41. DOI: 10.1186/s12958-021-00714-3 PMID: 33673853 DOI: 10.1186/s12958-021-00714-3

Zegers-Hochschild F, Adamson GD, Dyer S, Racowsky C, de Mouzon J, Sokol R, Rienzi L, Sunde A, Schmidt L, Cooke ID, Simpson JL, van der Poel S. The International Glossary on Infertility and Fertility Care, 2017. Hum Reprod. 2017;32:1786-801. DOI: 10.1093/humrep/dex234 PMID: 29117321 DOI: 10.1093/humrep/dex234 